\title{
Past, Present and Future Highlights in Top and Higgs Physics from the CDF experiment
}

\author{
M. C. Kruse (on behalf of the CDF collaboration) \\ Department of Physics, Duke University, Box 90305, Durham NC 27708
}

\begin{abstract}
The 1992-1995 running of the Fermilab Tevatron (the so-called Run 1) ended with many important physics goals accomplished, including the discovery of the top quark, and the anticipation of many further questions be to answered in the future. In March 2002, after many detector upgrades by both the CDF and D $\varnothing$ experiments, and significant upgrades of the accelerator itself, the Tevatron Run 2 began (after a detector commissioning run) with the ultimate goal of discovering the Higgs boson. Here, we will highlight some important Run 1 results from CDF in the areas of top quark and Higgs boson physics, show some preliminary studies from Run 2, and give some expectations of what Run 2 will ultimately provide to our understanding of matter.
\end{abstract}

\section{INTRODUCTION}

As yet there as been no direct evidence for physics beyond the standard model(SM), although there have been numerous tantalizing suggestions, both experimental and theoretical, that physics beyond our current understanding does exist and might be within our reach. After the success of the Fermilab Tevatron "Run 1" (1992 - 1995), operating with protons on antiprotons with a center of mass energy of $\sqrt{s}=1.8 \mathrm{TeV}$ and a bunch spacing of $3.6 \mu \mathrm{s}$, a shutdown ensued to upgrade the accelerator and the collider experiment detectors (CDF and DØ) for "Run 2", in order to expand our reach toward whatever new physics might exist.

The integrated luminosity from Run 1 was about $110 \mathrm{pb}^{-1}$, with typical instantaneous luminosities of $1.5 \times 10^{31} \mathrm{~cm}^{-2} \mathrm{~s}^{-1}$ (with the best instantaneous luminosity about double this). Run 2 will be split into 2 periods at a higher energy of $\sqrt{s}=2 \mathrm{TeV}$ : Run $2 \mathrm{a}$, which has now started (in March 2002), operating with a bunch spacing of 396ns, and Run $2 \mathrm{~b}$, which will begin in 2006 with a possible bunch spacing $132 \mathrm{~ns}$. The integrated luminosity from Run $2 \mathrm{a}$ is expected to be 20 times that of Run 1 at $2 \mathrm{fb}^{-1}$, and the projected total luminosity by the end of Run $2 b$ is $15 \mathrm{fb}^{-1}$, with the main objective of giving the Tevatron sufficient sensitivity to discover the SM Higgs boson (or a "SM-like" Higgs boson).

The CDF detector is designed for general purpose use, with a tracking system within a 1.4 T magnetic field, calorimetry, and a muon detection system. Figure 1 shows a schematic of the CDF Run $2 b$ detector. The tracking system consists of a silicon system (somewhat different in Run 1 and Run 2) for the precise measurement of secondary vertices from $b$-quark decays ( $c \tau=140 \mu \mathrm{m}$, which implies, with a boost factor of about $7, B$ hadrons travel on average about $3 \mathrm{~mm}$ before decaying); a central outer tracker (COT), consisting of wire chambers for the measurement of charged particle 

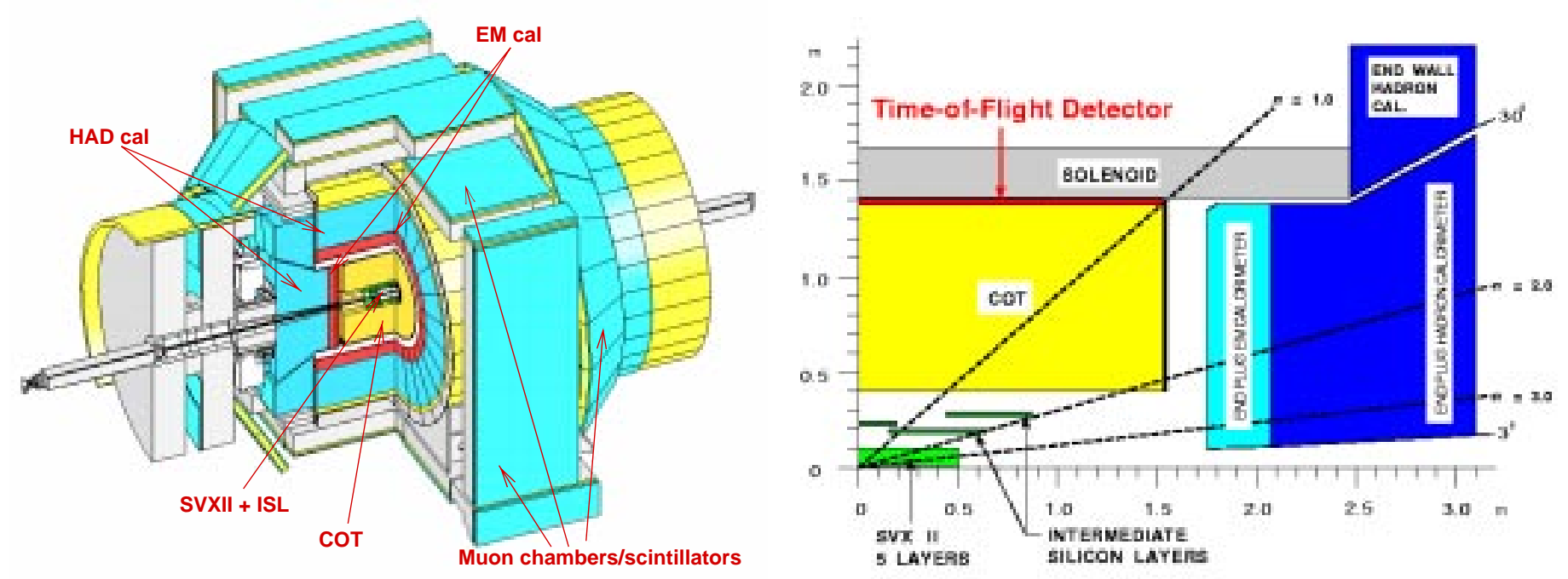

FIGURE 1. Schematic of the CDF Run 2 detector (left) and a side view of the tracking system (right).

momenta, and a time of flight (TOF) detector (new in Run 2) for a measurement of particle speed, which together with the momentum can identifying particles by their mass. Outside the magnetic solenoid lies the electromagnetic and hadronic calorimeters for the measurements of energy deposition. Electrons, photons, and jets, deposit almost all their energy in the calorimeters. Muons travel through the calorimeters depositing only a small fraction of their energy, and are detected by the muon chambers (gas chambers) which surround the calorimeters and steel absorbers. Momenta and Energies are measured most precisely in the plane transverse to the beam direction, therefore, typically only the transverse components, $P_{T}$ and $E_{T}$, are used in determining the selection criteria for particles.

In the following sections we will summarise a selection of past results using the CDF detector, and discuss what we expect in the next several years, concentrating on the physics involving the top quark, and searches for Higgs bosons.

\section{PAST HIGHLIGHTS: SELECTED RESULTS FROM RUN 1}

One of the successes from Run 1 was the discovery of the top quark, announced jointly by the CDF and D $\varnothing$ collaborations in 1995 . Everything we currently know about the top quark is based on only about $100 t \bar{t}$ events. In Table 1 we summarise the mass and cross section measurements from Run 1. At the Tevatron top quarks are produced predominantly in pairs via the strong interaction, with a cross section at $\sqrt{s}=1.8 \mathrm{TeV}$ of about $\sigma_{t \bar{t}}=5 \mathrm{pb}$, and at $\sqrt{s}=2.0 \mathrm{TeV}$ about $7 \mathrm{pb}$ [1]. The top quark can also be produced single via the electroweak interaction with about half the cross section, but with a final state that is difficult to extract from background processes. The top quark decays almost exclusively to $W b$, and it is the different combinations of $W$ decays that characterise the $t \bar{t}$ decay channels. The "dilepton" decay channel is that in which both $W$ 's decay 
TABLE 1. Summary of top quark properties as measured by the Tevatron from about $110 \mathrm{pb}^{-1}$ of data in Run 1 . The measured cross sections are shown for each decay channel of $t \bar{t}$ separately, and for the combination of channels. The top masses shown for each experiment are the results of combining the measurements in each decay channel.

\begin{tabular}{|c|ccc|}
\hline Property & CDF measurement & DØ measurement & Combined(CDF + DØ) \\
\hline Mass $\left(\mathrm{GeV} / c^{2}\right)$ & $176.1 \pm 6.6$ & $172.1 \pm 6.8$ & $174.3 \pm 5.1$ \\
$\sigma_{t \bar{t}}$ (dilepton) $(\mathrm{pb})$ & $8.4_{-3.5}^{+4.5}$ & $6.4 \pm 3.4$ & \\
$\sigma_{t \bar{t}}$ (lepton + jets) $(\mathrm{pb})$ & $5.7_{-1.5}^{+1.9}$ & $5.2 \pm 1.8$ & \\
$\sigma_{t \bar{t}}$ (all-hadronic) $(\mathrm{pb})$ & $7.6_{-2.7}^{+3.5}$ & $7.1 \pm 3.2$ & \\
$\sigma_{t \bar{t}}$ (combined) $(\mathrm{pb})$ & $6.5_{-1.4}^{+1.7}$ & $5.9 \pm 1.7$ & \\
\hline
\end{tabular}

leptonically and has a branching ratio of about $5 \%$ (we only consider $W$ decays to $e v$ and $\mu v)$. In the "lepton + jets" decay channel one $W$ decays leptonically and the other hadronically (the branching ratio is about $30 \%$ ), and in the "all-hadronic" decay channel both $W$ 's decay hadronically (the branching ratio is about $45 \%$ ). The dilepton channel is therefore characterised by 2 highly energetic leptons ( $e$ or $\mu$ with $P_{T}>20 \mathrm{GeV}$ ) from the $W$ decays, a large amount of "missing energy" from the $W$ decay neutrinos (denoted $\mathbb{E}_{T}$ ), and 2 "jets" from the fragmentation of the $2 b$ quarks. Similarly, the "lepton + jets" channel is characterised by one energetic lepton, large missing energy, and 4 "jets" (from the $2 b$ quarks and the hadronic $W$ decay), and the all-hadronic channel is, as its name suggests, characterised by 6 "jets" only.

To increase the signal to background ratio in the "lepton + jets" and "all-hadronic" decay channels, CDF made extensive use of the silicon vertex detector (SVX) [2], which could identify the secondary vertices from $b$ quark decays. $b$ quarks have a lifetime of about $1.2 \mathrm{ps}(c \tau \sim 450 \mu \mathrm{m})$, and so, with an average boost of about a factor of 7 , they travel on average about $3 \mathrm{~mm}$ before decaying. The high precision of the CDF SVX detector allowed an efficiency for identifying at least one of the $b$ quarks from $t \bar{t}$ decay of about $50 \%$ [4].

In addition, when $b$ quarks decay leptonically (branching ratio of about $20 \%$ for $e$ or $\mu$ ), one can "tag" the jet as coming from a $b$ quark by identifying the lepton in the jet (called an "SLT" tag). This has a much lower efficiency due to the difficulty in identifying these leptons and the low branching ratio of $b$ 's to leptons. For a $t \bar{t}$ event the efficiency of tagging at least one of the $b$ jets in this way was about $20 \%$.

The large mass of the top quark may be an indication of a special role in electroweak symmetry breaking (EWSB), or a window to other new physics. In Run 2 we will certainly be studying the top quark in a lot more detail, and following up on some interesting observations and searches from Run 1. Some of these include:

- The $e \mu$ top dilepton channel is particularly clean in that the only processes that can significantly contribute are $W W$ production, $Z \rightarrow \tau^{+} \tau^{-}$(where in both cases extra jets come from higher order processes), and $t \bar{t} \rightarrow e v \mu \nu b \bar{b}$ (the $e e$ and $\mu \mu$ channels have a large background from Drell-Yan production of dilepton events, something the $e \mu$ channel doesn't suffer from). In Run 1 the total expected background in the $e \mu$ channel was about 0.8 events, the expected contribution from $t \bar{t}$ was about 2.4 events (assuming $\sigma_{t \bar{t}} \sim 5 \mathrm{pb}$ ), and the observed number of events was 7 [3]. Al- 
though the observed excess over the SM prediction was not statistically significant, it was also noted that the kinematics of a couple of these events were difficult to reconcile with SM processes. (One $e e$ and one $\mu \mu$ top dilepton candidate events were also observed with an expected background of 1.7 events and $t \bar{t}$ expectation of 1.8 events.)

- In the "lepton + jets" channel, the $t \bar{t}$ production cross section was measured using lepton + missing energy events with 3 or more jets, at least one of which was $b$ tagged (by the SVX or SLT taggers) [4]. A detailed study of the heavy flavour content in all the jet multiplicities revealed an excess of events with 2 or 3 jets with a jet containing both a SVX and SLT $b$-tag. The 13 observed events over a SM expectation of $4.4 \pm 0.6$, prompted a careful study of event kinematics which were found to be very difficult to explain from expected SM distributions [5].

- Although production of top quark pairs was observed and used to measure top quark properties, production of single top was not observed in Run 1. The lower cross section (about $2.4 \mathrm{pb}$ ) and higher background rate (the signal is mostly in the $W+2$-jets sample) only allowed cross section limits to be set in Run 1 [6], however, this measurement will be important in Run 2, as it is a direct probe to the $W t b$ vertex, and therefore can be used for a direct measurement of $\left|V_{t b}\right|$.

- Studies of the $t \bar{t}$ invariant mass spectrum provide a general search for new heavy objects decaying to top pairs (for example, dynamical $t \bar{t}$ condensates in models such as "Topcolor" could be responsible for EWSB). In Run 1 we searched for such resonances in the $M_{t \bar{t}}$ spectrum [7]. In Figure 2 we show this distribution using events in the "lepton + jets" channel. This distribution was used to place limits on models predicting narrow $t \bar{t}$ resonances.

Within the SM mass is generated, and the electroweak symmetry is broken, by the Higgs mechanism. All the particles of the SM have been observed, except for the Higgs boson, so its discovery is currently one of the most important goals in high energy physics. Precision electroweak measurements (including the top mass) predict a light SM Higgs [8] of $88_{-35}^{+53} \mathrm{GeV} / c^{2}$. In Run $1 \mathrm{CDF}$ conducted searches for low mass Higgs bosons $\left(M_{H}<120 \mathrm{GeV}\right)$, using its associated production with vector bosons $\left(W^{ \pm}\right.$or $\left.Z^{0}\right)$. At such masses the Higgs decays predominantly to $b \bar{b}$. Therefore, even though the cross section for $g g \rightarrow H\left(0.7 \mathrm{pb}\right.$ at $\left.M_{H}=120 \mathrm{GeV}\right)$ is much larger than the cross sections for $W H$ and $Z H$ production $\left(0.16 \mathrm{pb}\right.$ and $0.10 \mathrm{pb}$ respectively at $M_{H}=120 \mathrm{GeV}$ ) [9], the Tevatron experiments are more sensitive to the latter production mechanisms, as single Higgs production is overwhelmed by an irreducible QCD di-jet background.

In Run 1 CDF searched for the SM Higgs in the following decay channels:

- $Z H \rightarrow \ell \ell b \bar{b}$ (with at least $1 b$-tagged jet): signal characterised by 2 highly energetic leptons $\left(P_{T}>20 \mathrm{GeV}\right)$ and 2 jets. Expected $3.2 \pm 0.7$ events (observed 5).

- $Z H \rightarrow v v b \bar{b}$ (with at least one $b$-tagged jet): signal characterised by large missing energy and 2 jets. Expected $39 \pm 4$ single $b$-tagged events (observed 40), and $3.9 \pm 0.6$ double $b$-tagged events (observed 4 ).

- $W H \rightarrow \ell v b \bar{b}$ (with at least one $b$-tagged jet): signal characterised by one energetic lepton, large missing energy and 2 jets. Expected $30 \pm 5$ single $b$-tagged events 

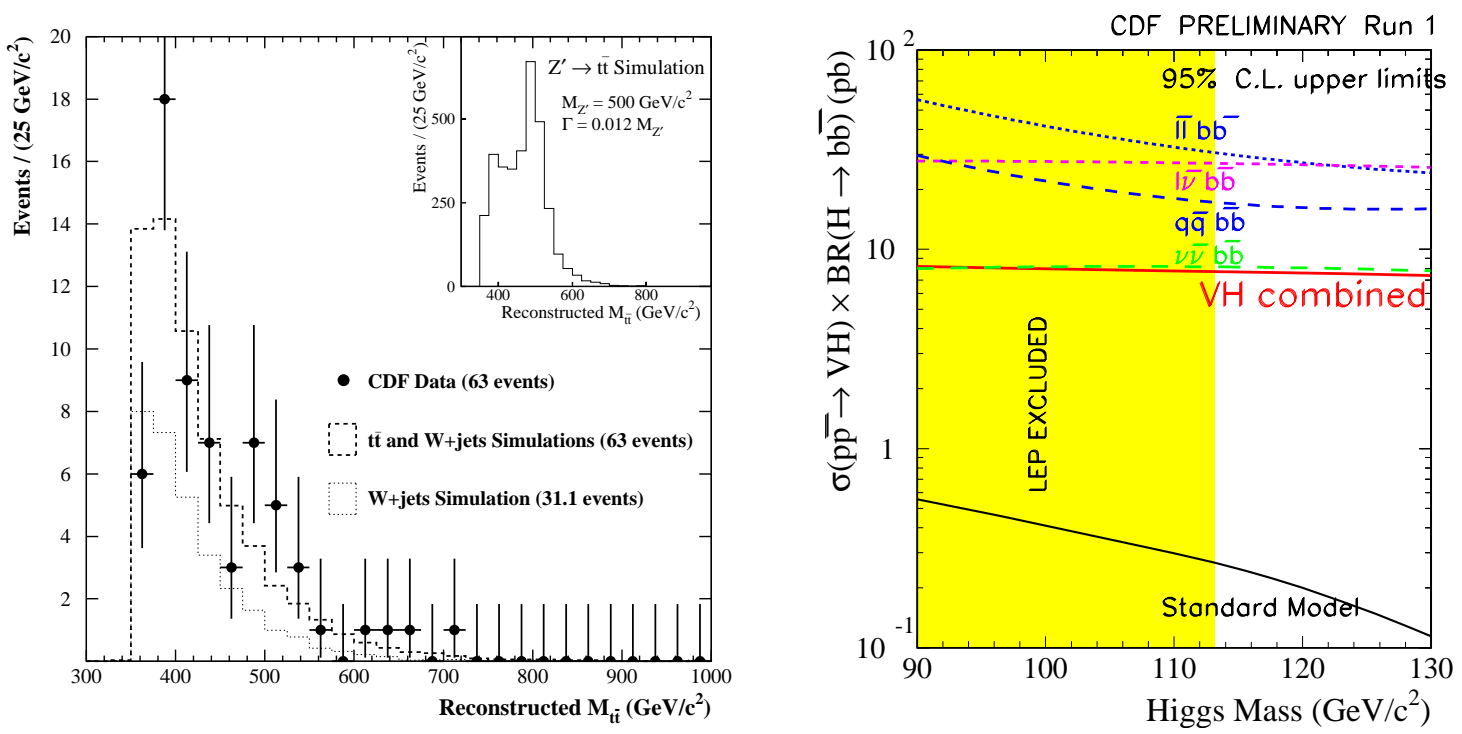

FIGURE 2. CDF Run 1 results from searches for $t \bar{t}$ resonances (left), and the SM Higgs boson (right). The $t \bar{t}$ invariant mass spectrum compares the CDF data to that expected from the SM. The inset shows the expected distribution from a narrow $500 \mathrm{GeV}$ resonance. The Run $195 \%$ confidence level limits for $W H+Z H$ production are shown on the right as a function of Higgs mass for the difference decay channels analysed. Also shown is the region excluded by LEP2.

(observed 36), and 3.0 \pm 0.6 double $b$-tagged events (observed 6).

- $W / Z H \rightarrow q q^{\prime} b \bar{b}$ (with at least two $b$-tagged jets): signal characterised by 4 jets. Expected 600 double $b$-tagged events (observed 589).

No signal was observed in the di-jet invariant mass spectra, therefore, cross section limits were determined by fitting the observed spectra to a combination of the expected di-jet spectra from background processes and from $W H$ and $Z H$ signal. Figure 2 summarises the CDF 95\% confidence level (CL) cross section limits for all the individual decay channels as well as the combined result, as a function of the Higgs mass. The limits are about 30 times higher than the SM predictions, which sets the scale for the luminosity needed in order to observe the SM Higgs boson in Run 2.

\section{PRESENT HIGHLIGHTS: THE BEGINNING OF RUN 2}

For Run 2a the Fermilab accelerator complex underwent a major upgrade; the most significant components being a new storage ring (the "Main Injector") to replace the "Main Ring" as the supplier of high energy protons and antiprotons to the Tevatron; and the "Recycler", an additional new ring housed in the same Main Injector tunnel which will allow the reusing of antiprotons at the end of a store, which otherwise typically take several hours to accumulate and cool. The Recycler is expected to come online in October 2004 in the more limited capacity as an antiproton accumulator, and will not be fully operational to reuse antiprotons until the following year. The Run 2a instantaneous luminosity goal is $5-8 \times 10^{31} \mathrm{~cm}^{-2} \mathrm{~s}^{-1}$ before the Recycler is fully operational, and $2 \times 10^{32} \mathrm{~cm}^{-2} \mathrm{~s}^{-1}$ afterward. Figure 3 shows the progress of achieved instantaneous luminosities toward this goal, up until January 2003. 


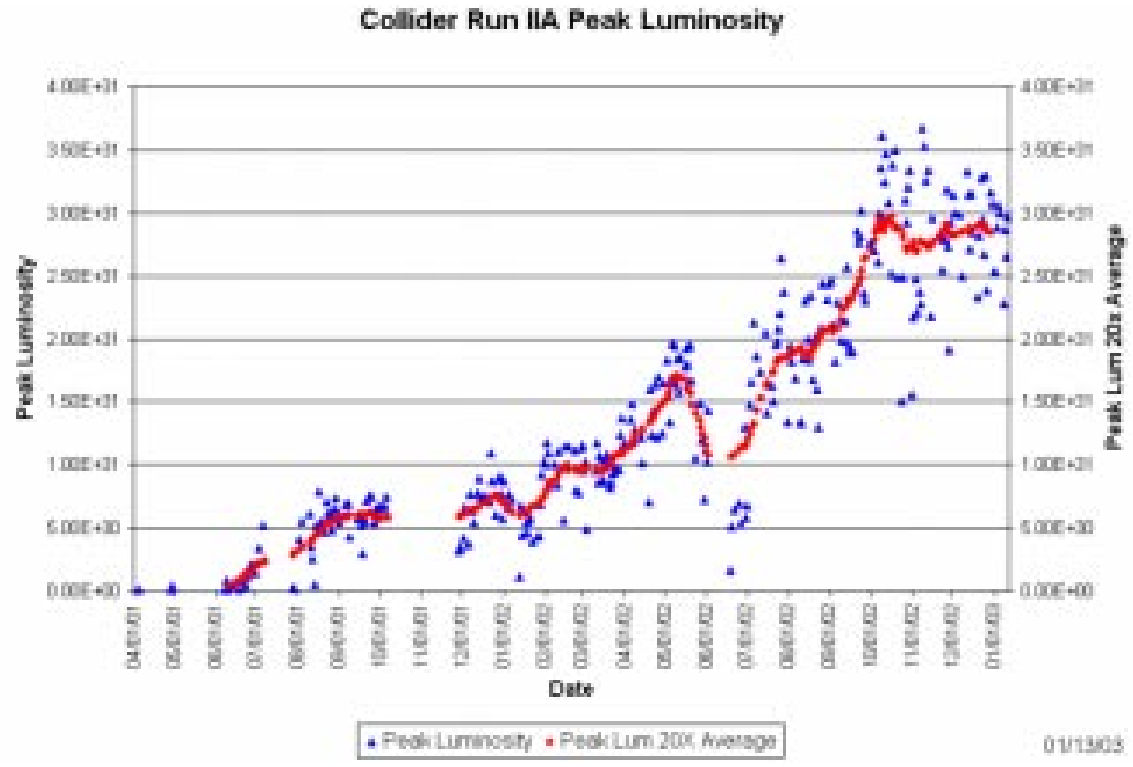

FIGURE 3. Initial luminosity achieved by the accelerator versus time in Run $2 \mathrm{a}$. The stores up to January 2002 were used for CDF detector commissioning. The gaps in luminosity represent shutdowns. The average of the 20 previous stores is also plotted.

As of mid-January 2003, the accelerator has delivered about $150 \mathrm{pb}^{-1}$ of integrated luminosity, with about $100 \mathrm{pb}^{-1}$ being written to tape. Of that, CDF currently has about $70 \mathrm{pb}^{-1}$ of good data for analysis (this ratio has recently increased due to better performance and understanding of the Run $2 \mathrm{CDF}$ detector). The baseline goal of the accelerator through to October 2003 is to deliver $10 \mathrm{pb}^{-1}$ per week, translating to an expected data sample of about $200 \mathrm{pb}^{-1}$ of good data by October 2003, about twice the integrated luminosity of Run 1.

The Run 2 CDF detector upgrades [10] mostly included upgrades to the Run 1 tracking system (Figure 1 shows a side view of the Run 2 tracking system), including a new silicon system, a new drift chamber (the "COT"), and a Time-of-flight detector. The new silicon system consists of a layer of silicon on the beam pipe ("layer 00"), a 5layer silicon detector (the "SVX 2", which replaces the 4-layer Run 1 SVX), and 2 outer layers of silicon (the "ISL"). This system will provide improved $b$-tagging capabilities, important for both top and Higgs analyses. This system is currently working well in Run 2 , after a considerable time required for commissioning.

Of significant impact to the physics capabilities in Run 2, is a secondary vertex trigger (the "SVT"), which selects tracks of at least $2 \mathrm{GeV}$ and requires they have an impact parameter (the minimum transverse distance from the primary vertex to the extrapolated track) of at least $100 \mu \mathrm{m}$. This trigger will provide huge samples for beauty and charm quark physics (expect $\sim 10^{7}$ fully reconstructed charm decays in $2 \mathrm{fb}^{-1}$ ), revolutionizing heavy flavour physics at hadron colliders. The SVT is currently working well with an impact parameter resolution less than $50 \mu \mathrm{m}$ (which includes a $\sim 30 \mu \mathrm{m}$ beam spot resolution). Also, of importance for $b$-jet calibrations to top and Higgs analyses, is the large $Z \rightarrow b \bar{b}$ sample this trigger will provide. 
TABLE 2. Summary of expected top production numbers at the Tevatron, comparing Run 1 with Run 2 . The expected observed numbers in the various decay channels (the last 4 rows) use the expected efficiencies for observing top events at CDF.

\begin{tabular}{|l|ccc|}
\hline & Run 1 & Run 2a & Run 2b \\
\hline CM Energy (TeV) & 1.8 & 2.0 & 2.0 \\
Peak Luminosity $\left(\mathrm{cm}^{-2} \mathrm{~s}^{-1}\right)$ & $2 \times 10^{31}$ & $2 \times 10^{32}$ & $1 \times 10^{33}$ \\
Integrated Luminosity $\left(\mathrm{fb}^{-1}\right)$ & 0.11 & 2.0 & 15.0 \\
& & & \\
$\sigma(t \bar{t})(p b)$ & 5.0 & 7.0 & 7.0 \\
$\sigma($ single top) & 2.5 & 3.4 & 3.4 \\
& & & \\
Number of $(t \bar{t})$ produced & 500 & 14000 & 210000 \\
Number of single top produced & 250 & 7000 & 100000 \\
& & & \\
$N\left(t \bar{t} \rightarrow \ell^{+} \ell^{-}+\mathbb{E}_{T}+2\right.$ jets $)(“ d i l e p t o n ”$ channel $)$ & 4 & 150 & 2200 \\
$N(t \bar{t} \rightarrow \ell+\geq 3 j)(\geq 1 b$-tag) & 25 & 1400 & 20000 \\
$N(t \bar{t} \rightarrow \ell+\geq 4 j)(2 b$-tags) & 5 & 600 & 9000 \\
$N($ single $\mathrm{t})(W+2$ jets sample with $1 b$-tag) & 3 & 140 & 2000 \\
\hline
\end{tabular}

We will present the first preliminary top physics results from Run 2 in March 2003. These will include $t \bar{t}$ cross sections in both the "dilepton" and "lepton + jets" channels. Recall the dilepton channel showed an interesting excess in Run 1, so results from this channel will be eagerly awaited.

\section{FUTURE HIGHLIGHTS: EXPECTATIONS FROM RUN 2}

Before we have a realistic chance of discovering the Higgs boson, we will take advantage of the fact that the Tevatron will remain the only source of top quarks for the next several years. Using the expected top quark samples summarised in Table 2 we will precisely measure many top quark parameters and use top as a possible window to new physics (as eluded to above). By the end of Run $2 \mathrm{a}$ alone $\left(2 \mathrm{fb}^{-1}\right)$, we should measure the top quark mass to less than $2 \%$ precision and the total cross section to less than $7 \%$ precision. In addition, the precision on the cross section for single top production should be around $20 \%$ (and $8 \%$ after Run 2 b).

The light SM Higgs predicted by precision electroweak measurements suggests this elusive final component of the SM might be possible to discover at the Tevatron in the next few years. However, even with the significant improvements to the Tevatron to increase the luminosity, this important goal will not be easy. In addition to the needed increase in luminosity, an improved di-jet mass resolution will be critical to the sensitivity for observing a $H \rightarrow b \bar{b}$ signal over the non-resonant di-jet mass from background processes (mostly $W+$ jets). In Run 1 we observed $Z \rightarrow b \bar{b}$ with low statistics [11], but in Run 2 with the new SVT trigger, we will have a large $Z \rightarrow b \bar{b}$ sample from which to gain a detailed understanding of $b$-jet energy corrections, and hence improve the $b \bar{b}$ invariant mass resolution. For maximal sensitivity for a Tevatron Higgs discovery, $\mathrm{CDF}$ and $\mathrm{D} \emptyset$ will need to combine their results over all channels. If 
so, then with $\sim 10 \mathrm{fb}^{-1}$ the Higgs could be discovered at the Tevatron (at the $3 \sigma$ level) if $M_{H}<130 \mathrm{GeV} / c^{2}$, or excluded (at the $95 \% \mathrm{CL}$ limit) up to about $180 \mathrm{GeV} / c^{2}$ [12]. The upgrades for Run $2 b$ [13] are therefore imperative for a realistic chance to discover a SM (or "SM-like") Higgs boson at the Tevatron.

\section{SUMMARY}

The Run 2 upgrades to the CDF detector, and to the Fermilab accelerator complex, greatly enhance the physics possibilities at CDF. In the short term (the next year or two), some interesting Run 1 top physics curiosities should be resolved and single top production should be confirmed. The longer term goals for Run 2 include the discovery the Higgs boson(s), and precision measurements of top quark properties, which may open a window to new physics given its uniquely large mass and possibility for a role in electroweak symmetry breaking. These goals, in addition to many other important Run 2 physics endeavours not covered here, will well position the Tevatron to making a huge impact on our understanding of matter this decade.

\section{ACKNOWLEDGMENTS}

I would like to thank the Division of Particles and Fields of the Mexican Physical Society for the opportunity to participate in a stimulating and well organised X Mexican School of Particles and Fields. This work was supported by the U.S. Department of Energy and National Science Foundation; the Italian Istituto Nazionale di Fisica Nucleare; the Ministry of Education, Science and Culture of Japan; the Natural Sciences and Engineering Research Council of Canada; the National Science Council of the Republic of China; and the A.P. Sloan Foundation.

\section{REFERENCES}

1. E. Laenen, J. Smith, W.L. van Neerven, Phys. Lett. B 321, 254-258, 1994;

R. Bonciani, S. Catani, M.L. Mangano, P. Nason, Nucl. Phys. B 529, 424, 1998.

2. D. Amidei et al., Nucl. Inst. Meth. Phys. Res., A 360, 137, 1995.

3. F. Abe et al., Phys. Rev. Lett., 80, 2779-2784, 1998.

4. T. Affolder et al., Phys. Rev. D64, 032002 (2001).

5. D. Acosta et al., Phys. Rev. D65, 52007 (2002);

G. Apollinari et al., Phys. Rev. D65, 032004 (2002).

6. D. Acosta et al., Phys. Rev. D65, 091102, 2002.

7. T. Affolder et al., Phys. Rev. Lett., 85, 2062-2067, 2000.

8. The LEP electroweak working group: http: / / lepewwg • web. cern. ch/LEPEWWG/

9. M. Spira, hep-ph/9810289.

10. CDF Collaboration, “The CDF II Detector Technical Design Report”, FERMILAB-Pub-96/390-E.

11. T. Dorigo (CDF collaboration), hep-ex/9806022.

12. M. Carena, J. Conway, H. Haber, J. Hobbs at al., Report of the Tevatron Higgs Working Group, hep-ph/0010338.

13. The CDF Run 2b upgrade: http://www-cdf . fnal .gov/run2b.html 